Bull. Korean Math. Soc. 42 (2005), No. 3, pp. 491-499

\title{
q-ANALOGUE OF EULER-BARNES' NUMBERS AND POLYNOMIALS
}

\author{
Leechae Jang and TaEkyun Kim
}

\begin{abstract}
Recently, Kim[2,6] has introduced an interesting EulerBarnes' numbers and polynomials. In this paper, we construct the $q$-analogue of Euler-Barnes' numbers and polynomials, and investigate their properties.
\end{abstract}

\section{Introduction}

Let $w, a_{1}, a_{2}, \cdots, a_{r}$ be complex numbers such that $a_{i}(\neq 0)$ for each $i=1,2, \cdots, r$. Then the Euler-Barnes' polynomials of $w$ with parameters $a_{1}, a_{2}, \cdots, a_{r}$ are defined as

$$
\frac{(1-u)^{r}}{\prod_{j=1}^{r}\left(e^{a_{j} t}-u\right)} e^{w t}=\sum_{n=0}^{\infty} H_{n}^{(r)}\left(w, u \mid a_{1}, a_{2}, \cdots, a_{r}\right) \frac{t^{n}}{n !},
$$

for $u \in \mathbb{C}$ with $|u|>1$, cf.[6]. In the special case $w=0$, the above polynomials are called the $r$-th Euler-Barnes' numbers. We write

$$
H_{n}^{(r)}\left(u \mid a_{1}, a_{2}, \cdots, a_{r}\right)=H_{n}^{(r)}\left(0, u \mid a_{1}, a_{2}, \cdots, a_{r}\right) .
$$

Throughout this paper, the symbols $\mathbb{Z}, \mathbb{Z}_{p}, \mathbb{Q}_{p}, \mathbb{C}$ and $\mathbb{C}_{p}$ will respectively denote the ring of rational integers, the ring of $p$-adic integers, the field of $p$-adic numbers, the complex number field and the completion of algebraic closure of $\mathbb{Q}_{p}$. Let $\nu_{p}$ be the normalized exponential valuation of $\mathbb{C}_{p}$ with $|p|_{p}=p^{-\nu_{p}(p)}=p^{-1}$. When one talks of $q$-extension, $q$ is variously considered as an indeterminate, a complex number $q \in \mathbb{C}$, or $p$-adic number $q \in \mathbb{C}_{p}$, cf. [2-5]. If $q \in \mathbb{C}$, one normally assumes

Received March 2, 2004.

2000 Mathematics Subject Classification: 11B68.

Key words and phrases: Euler numbers, Bernoulli numbers, zeta function.

This paper was supported by Jangjeon Mathematical Society. 
$|q|<1$. If $q \in \mathbb{C}_{p}$, one normally assumes $|1-q|_{p} \leq p^{-\frac{1}{p-1}}$, so that $q^{x}=\exp (x \log q)$. In this paper we use the notation:

$$
[x]=[x: q]=\frac{1-q^{x}}{1-q}, \text { cf. }[1,2,8] .
$$

The ordinary Euler numbers $E_{m}$ are defined by the generating function in the complex number field as

$$
\frac{2}{e^{t}+1}=\sum_{m=0}^{\infty} E_{m} \frac{t^{m}}{m !}, \quad(|t|<\pi), \text { cf. [9]. }
$$

Let $u$ be an algebraic in complex number field. Then Frobenius-Euler numbers are defined as

$$
\frac{1-u}{e^{t}-u}=\sum_{n=0}^{\infty} H_{n}(u) \frac{t^{n}}{n !}, \quad(|t|<\pi), \quad \text { cf. }[9,10] .
$$

Note that $H_{n}(-1)=E_{n}$. Also, Carlitz defined the $q$-analogue of Frobenius-Euler numbers and polynomials as follows:

$$
H_{0}(u: q)=1,(q H+1)^{k}-u H_{k}(u: q)=0 \text { if } k \geq 1,
$$

where $u$ is a complex number with $|u|>1$ :

$$
H_{k}(u, x: q)=\left(q^{x} H+[x]\right)^{k} \text { if } k \geq 0, \text { cf. [2,11], }
$$

with the usual convention about replacing $H^{k}(u: q)$ by $H_{k}(u: q)$. For any positive integer $N, z \in \mathbb{C}_{p}$,

$$
\mu_{z}\left(a+p^{N} \mathbb{Z}_{p}\right)=\frac{z^{a}}{\left[p^{N}: z\right]}
$$

can be extended to distribution on $\mathbb{Z}_{p}$, cf. $[1,2,7,13]$. Let $U D\left(\mathbb{Z}_{p}\right)$ be denoted by the set of uniformly differentiable functions on $\mathbb{Z}_{p}$. Then this distribution admits the following integral for $f \in U D\left(\mathbb{Z}_{p}\right)$ :

$$
I_{z}(f)=\int_{\mathbb{Z}_{p}} f(x) d \mu_{z}(x)=\lim _{N \rightarrow \infty} \frac{1}{\left[p^{N}: z\right]} \sum_{x=0}^{p^{N}-1} f(x) z^{x}, \text { cf. }[1,2,12] .
$$

The purpose of this paper is to construct the $q$-analogue of EulerBarnes' numbers and investigate their properties. 


\section{2. $q$-analogue of multiple Euler numbers and polynomials}

Let $d$ be a fixed integer and let $p$ be a fixed prime number. We set

$$
\begin{aligned}
X & =\underset{\varliminf}{\varliminf_{N}}\left(\mathbb{Z} / d p^{N} \mathbb{Z}\right), \\
X^{*} & =\bigcup_{\substack{0<a<d p \\
(a, p)=1}} a+d p \mathbb{Z}_{p}, \\
a+d p^{N} \mathbb{Z}_{p} & =\left\{x \in X \mid x \equiv a\left(\bmod d p^{N}\right)\right\},
\end{aligned}
$$

where $a \in \mathbb{Z}$ lies in $0 \leq a<d p^{N}$.

Let $u \in \mathbb{C}_{p}$ with $\left|1-u^{f}\right|_{p} \geq 1$ for each positive integer $f$ and let $a_{1}, a_{2}, \cdots, a_{r}$ be non-zero $p$-adic integers. For $w \in \mathbb{Z}_{p}$, we consider the $q$-analogue of Euler-Barnes' polynomials by using $p$-adic invariant integrals as follows: For $q \in \mathbb{C}_{p}$ with $|1-q|_{p}<p^{-\frac{1}{1-p}}$, define

$$
\begin{aligned}
& H_{n}^{(r)}\left(w, u, q \mid a_{1}, a_{2}, \cdots, a_{r}\right) \\
= & \underbrace{\int_{\mathbb{Z}_{p}} \cdots \int_{\mathbb{Z}_{p}}}_{r \text { times }}\left[w+\sum_{j=1}^{r} a_{j} x_{j}: q\right]^{n} d \mu_{u}\left(x_{1}\right) \cdots d \mu_{u}\left(x_{r}\right) .
\end{aligned}
$$

By (1), we note that

$$
\begin{aligned}
& \underbrace{\int_{\mathbb{Z}_{p}} \cdots \int_{\mathbb{Z}_{p}}}_{r \text { times }}\left[w+\sum_{j=1}^{r} a_{j} x_{j}: q\right]^{n} d \mu_{u}\left(x_{1}\right) \cdots d \mu_{u}\left(x_{r}\right) \\
= & \lim _{N \rightarrow \infty} \frac{1}{\left[p^{N}: u\right]^{r}} \sum_{x_{1}, \cdots, x_{r}=0}^{p^{N}-1}\left[w+\sum_{j=1}^{r} a_{j} x_{j}: q\right]^{n} u^{\sum_{j=1}^{r} x_{j}} \\
= & \lim _{N \rightarrow \infty}\left(\frac{1-u}{1-u^{p^{N}}}\right)^{r} \\
& \times \sum_{x_{1}, \cdots, x_{r}=0}^{p^{N}-1}\left(\sum_{l=0}^{n}\left(\begin{array}{l}
n \\
l
\end{array}\right)\left(\frac{1}{1-q}\right)^{n}(-1)^{l} q^{l\left(w+\sum_{j=1}^{r} a_{j} x_{j}\right)} u^{\sum_{j=1}^{r} x_{j}}\right) \\
= & \frac{(1-u)^{r}}{(1-q)^{n}} \sum_{l=0}^{n}\left(\begin{array}{l}
n \\
l
\end{array}\right)(-1)^{l} q^{l w}\left(\frac{1}{\prod_{j=1}^{r}\left(1-q^{l a_{j}} u\right)}\right),
\end{aligned}
$$

where $\left(\begin{array}{l}n \\ l\end{array}\right)$ is binomial coefficient. Therefore we obtain the following: 
Theorem 1. For $n \geq 0$, we have

$$
\begin{aligned}
& H_{n}^{(r)}\left(w, u, q \mid a_{1}, \cdots, a_{r}\right) \\
= & \frac{(1-u)^{r}}{(1-q)^{n}} \sum_{l=0}^{n}\left(\begin{array}{l}
n \\
l
\end{array}\right)(-1)^{l} q^{l w}\left(\frac{1}{\prod_{j=1}^{r}\left(1-q^{l a_{j}} u\right)}\right) .
\end{aligned}
$$

Moreover,

$$
\lim _{q \rightarrow 1} H_{n}^{(r)}\left(w, u, q \mid a_{1}, \cdots, a_{r}\right)=H_{n}^{(r)}\left(w, u^{-1} \mid a_{1}, \cdots, a_{r}\right) .
$$

REMARK. (1) In the special case $w=0$, we write

$$
H_{n}^{(r)}\left(u, q \mid a_{1}, \cdots, a_{r}\right)=H_{n}^{(r)}\left(0, u, q \mid a_{1}, \cdots, a_{r}\right) .
$$

(2) Note that $\lim _{q \rightarrow 1} H_{n}^{(1)}(u, q \mid 1)=H_{n}\left(u^{-1}\right)$, cf. [8,9].

Let $G_{q}^{(r)}\left(t, u \mid a_{1}, a_{2}, \cdots, a_{r}\right)$ be the generating function of $H_{n}^{(r)}(u, q \mid$ $\left.a_{1}, \cdots, a_{r}\right)$ :

$$
G_{q}^{(r)}\left(t, u \mid a_{1}, \cdots, a_{r}\right)=\sum_{k=0}^{\infty} H_{k}^{(r)}\left(u, q \mid a_{1}, \cdots, a_{r}\right) \frac{t^{k}}{k !},
$$

for $q \in \mathbb{C}_{p}$ with $|1-q|_{p}<1, u \in \mathbb{C}_{p}$ with $\left|1-u^{f}\right|_{p} \geq 1$. Then we have

$$
\begin{aligned}
& G_{q}^{(r)}\left(t, u \mid a_{1}, \cdots, a_{r}\right) \\
= & \sum_{k=0}^{\infty} H_{k}^{(r)}\left(u, q \mid a_{1}, \cdots, a_{r}\right) \frac{t^{k}}{k !} \\
= & \sum_{k=0}^{\infty} \frac{(1-u)^{r}}{(1-q)^{k}} \sum_{i=0}^{k}\left(\begin{array}{c}
k \\
i
\end{array}\right)(-1)^{i}\left(\prod_{l=1}^{r} \frac{1}{1-q^{i a_{l}} u}\right) \frac{t^{k}}{k !} \\
= & (1-u)^{r} e^{\frac{t}{1-q}} \sum_{j=0}^{\infty}\left(\prod_{l=1}^{r} \frac{1}{1-q^{j a_{l}} u}\right)\left(\frac{1}{1-q}\right)^{j} \frac{t^{j}}{j !} .
\end{aligned}
$$

Therefore we obtain the following:

Theorem 2. For $q \in \mathbb{C}_{p}$ with $|1-q|_{p}<1, u \in \mathbb{C}_{p}$ with $\left|1-u^{f}\right|_{p} \geq 1$, we have

$$
G_{q}^{(r)}\left(t, u \mid a_{1}, \cdots, a_{r}\right)=e^{\frac{t}{1-q}}(1-u)^{r} \sum_{j=0}^{\infty}\left(\prod_{l=1}^{r} \frac{1}{1-q^{j a_{l}} u}\right)\left(\frac{1}{1-q}\right)^{j} \frac{t^{j}}{j !} .
$$


Corollary 3. For $q \in \mathbb{C}_{p}$ with $|1-q|_{p}<1, u \in \mathbb{C}_{p}$ with $|1-u|_{p} \geq 1$, we have

$$
\begin{aligned}
& G_{q}^{(r)}\left(x, t, u \mid a_{1}, \cdots, a_{r}\right) \\
= & \sum_{n=0}^{\infty} H_{n}^{(r)}\left(x, u, q \mid a_{1}, \cdots, a_{r}\right) \frac{t^{n}}{n !} \\
= & e^{\frac{t}{1-q}}(1-u)^{r} \sum_{j=0}^{\infty}\left(\prod_{l=1}^{r} \frac{1}{1-q^{j a_{l}} u}\right)\left(\frac{1}{1-q}\right)^{j} q^{j x} \frac{t^{j}}{j !} .
\end{aligned}
$$

Note that

$$
\lim _{q \rightarrow 1} G_{q}^{(r)}\left(x, t, u \mid a_{1}, \cdots, a_{r}\right)=\frac{\left(1-u^{-1}\right)^{r}}{\prod_{l=1}^{r}\left(e^{a_{j} t}-u^{-1}\right)} e^{x t} .
$$

By (1), the Euler-Barnes' polynomials of $x$ can be rewritten as $H_{n}^{(r)}\left(w, u, q \mid a_{1}, \cdots, a_{r}\right)=\sum_{k=0}^{n}\left(\begin{array}{l}n \\ k\end{array}\right)[w: q]^{n-k} q^{w k} H_{k}^{(r)}\left(u, q \mid a_{1}, \cdots, a_{r}\right)$.

From the above Eq.(1), we have the distribution relation for the $q$ analogue of Euler-Barnes'polynomials as follows:

ThEOREM 4. For $f \in \mathbb{N}$, we have

$$
\begin{aligned}
& \frac{1}{(u-1)^{r}} H_{n}^{(r)}\left(w, u, q \mid a_{1}, \cdots, a_{r}\right) \\
=[f: q]^{n} \sum_{i_{1}, \cdots, i_{r}=0}^{f-1} & \frac{u^{\sum_{j=1}^{r} i_{j}}}{\left(u^{f}-1\right)^{r}} \\
& \quad \times H_{n}^{(r)}\left(\frac{w+\sum_{j=1}^{r} a_{j} i_{j}}{f}, u^{f}, q^{f} \mid a_{1}, \cdots, a_{r}\right) .
\end{aligned}
$$

For $k \geq 0, f \in \mathbb{N}$, we set

(3) $E_{u: a_{1}, q}^{(k)}\left(x+f p^{k} \mathbb{Z}_{p}\right)=\frac{\left[f p^{N}: q\right]^{k} u^{x}}{1-u^{f p^{N}}} H_{k}^{(1)}\left(\frac{a_{1} x}{f p^{N}}, u^{f p^{N}}, q^{f p^{N}} \mid a_{1}\right)$

and this can be extended to a distribution on $X$. We show that $E_{u: a_{1}, q}^{(k)}$ is a distribution on $X$. For this, it suffices to check that

$$
\sum_{i=0}^{p-1} E_{u: a_{1}, q}^{(k)}\left(x+i f p^{N}+f p^{N+1} \mathbb{Z}_{p}\right)=E_{u: a_{1}, q}^{(k)}\left(x+f p^{k} \mathbb{Z}_{p}\right)
$$


By (2), we easily see that

$$
\begin{aligned}
& \sum_{i=0}^{p-1} \frac{\left[p: q^{f p^{N}}\right]^{k}}{1-\left(u^{f p^{N}}\right)^{p}}\left(u^{f p^{N}}\right)^{i} H_{k}^{(1)}\left(\frac{\frac{a_{1} x}{f p^{N}}+i a_{1}}{p},\left(u^{f p^{N}}\right)^{p},\left(q^{f p^{N}}\right)^{p} \mid a_{1}\right) \\
= & \frac{1}{1-u^{f p^{N}}} H_{k}^{(1)}\left(\frac{a_{1} x}{f p^{N}}, u^{f p^{N}}, q^{f p^{N}} \mid a_{1}\right) .
\end{aligned}
$$

Therefore, we have

(4)

$$
\begin{aligned}
& \sum_{i=0}^{p-1} E_{u: a_{1}, q}^{(k)}\left(x+i f p^{N}+f p^{N+1} \mathbb{Z}_{p}\right) \\
= & \sum_{i=0}^{p-1} \frac{\left[f p^{N+1}: q\right]^{k} u^{\left(x+i f p^{N}\right)}}{1-u^{f p^{N+1}}} \\
& \quad \times H_{k}^{(1)}\left(\frac{a_{1}\left(x+i f p^{N}\right)}{f p^{N+1}}, u^{f p^{N+1}}, q^{f p^{N+1}} \mid a_{1}\right) \\
= & u^{x} \sum_{i=0}^{p-1} \frac{\left[f p^{N}: q\right]^{k}\left[p: q^{f p^{N}}\right]^{k}}{1-\left(u^{f p^{N}}\right)^{p}}\left(u^{f p^{N}}\right)^{i} \\
& \times H_{k}^{(1)}\left(\frac{\frac{a_{1} x}{f p^{N}}+i a_{1}}{p},\left(u^{f p^{N}}\right)^{p},\left(q^{f p^{N}}\right)^{p} \mid a_{1}\right) \\
= & \frac{u^{x}\left[f p^{N}: q\right]^{k}}{1-u^{f p^{N}}} H_{k}^{(1)}\left(\frac{a_{1} x}{f p^{N}}, u^{f p^{N}}, q^{f p^{N}} \mid a_{1}\right) \\
= & E_{u: a_{1}, q}^{(k)}\left(x+f p^{k} \mathbb{Z}_{p}\right) .
\end{aligned}
$$

Next we show that $\left|E_{u: a_{1}, q}^{(k)}\right|_{p} \leq 1$. Indeed,

(5)

$$
\begin{aligned}
& E_{u: a_{1}, q}^{(k)}\left(x+f p^{N} \mathbb{Z}_{p}\right) \\
= & \sum_{i=0}^{k}\left(\begin{array}{c}
k \\
i
\end{array}\right)\left(\frac{u^{x}}{1-u^{f p^{N}}}\right)\left[a_{1} x: q\right]^{k-i}\left[f p^{N}: q\right]^{i} q^{a_{1} x i} \\
& \times H_{i}^{(1)}\left(u^{f p^{N}}, q^{f p^{N}} \mid a_{1}\right) .
\end{aligned}
$$

By induction on $i$, we see that

$$
\left|\frac{u^{x}}{1-u^{f p^{N}}} H_{i}^{(1)}\left(u^{f p^{N}}, q^{f p^{N}} \mid a_{1}\right)\right|_{p} \leq 1, \text { for all } i,
$$


where we use the assumption $\left|1-u^{f}\right|_{p} \geq 1$, it follows that we have

$$
\left|E_{u: a_{1}, q}^{(k)}\left(x+i f p^{N}+f p^{N} \mathbb{Z}_{p}\right)\right|_{p} \leq 1
$$

Thus $E_{u: a_{1}, q}^{(k)}$ is a measure on $X$. This measure yields an integral for each non-negative integers $k$ as follows:

Proposition 5. For $k \geq 0$, we have

$$
\int_{X} d E_{u: a_{1}, q}^{(k)}(x)=\int_{\mathbb{Z}_{p}} d E_{u: a_{1}, q}^{(k)}=\frac{1}{1-u} H_{k}^{(1)}\left(u, q \mid a_{1}\right) .
$$

It is easy to see that

$$
H_{0}\left(u, q \mid a_{1}\right)=1 .
$$

We may now mention the following formula which is easy to prove by (5) and (6):

$$
E_{u: a_{1}, q}^{(k)}\left(x+f p^{N} \mathbb{Z}_{p}\right)=\left[a_{1} x: q\right]^{k} \frac{u^{k}}{1-u^{f p^{N}}}+\left[f p^{N}: q\right] \times(p \text {-integral }) .
$$

Hence, we obtain the following :

$$
\begin{aligned}
\int_{X} d E_{u: a_{1}, q}^{(k)}(x) & =\frac{1}{1-u} \int_{X}\left[a_{1} x: q\right]^{k} d \mu_{u}(x) \\
& =\frac{1}{1-u} H_{k}^{(1)}\left(u, q \mid a_{1}\right) .
\end{aligned}
$$

From the above definition, we have the following:

ThEOREM 6. Let $a_{1}, a_{2}, \cdots, a_{r}$ be $p$-adic integers. Then we obtain:

$$
\begin{aligned}
& \left(\frac{1}{1-u}\right)^{r} H_{k, \chi}^{(r)}\left(u, q \mid a_{1}, \cdots, a_{r}\right) \\
= & \frac{1}{\left(1-u^{d}\right)^{r}}[d: q]^{k} \sum_{i_{1}, \cdots, i_{r}=0}^{d-1} u^{\sum_{j=1}^{r} i_{j}}\left(\prod_{j=1}^{r} \chi\left(i_{j}\right)\right) \\
& \times H_{k}^{(r)}\left(\frac{\sum_{j=1}^{r} a_{j} i_{j}}{d}, u^{d}, q^{d} \mid a_{1}, \cdots, a_{r}\right) .
\end{aligned}
$$


Note that

$$
\int_{X} \chi(x) d E_{u: a_{1}, q}^{(k)}(x)=\frac{1}{1-u} H_{k, \chi}^{(1)}\left(u, q \mid a_{1}\right) .
$$

Let $\omega$ be denoted as the Teichmuller character $\bmod p$ (if $p=2$, $\bmod 4)$. For $x \in X^{*}$, we set

$$
\langle x: q\rangle=\frac{[x: q]}{w(x)}
$$

Note that $|\langle x: q\rangle-1|_{p}\left\langle p^{-\frac{1}{p-1}},\langle x: q\rangle^{s}\right.$ is defined as $\exp \left(s \log _{p}\langle x: q\rangle\right)$ for $|s|_{p} \leq 1$. For $s \in \mathbb{Z}_{p}$, define

$$
L_{p, q: a_{1}}(u \mid s, \chi)=\int_{X^{*}}\left\langle a_{1} x: q\right\rangle^{-s} \chi(x) d \mu_{u}(x)
$$

Then we have

$$
\begin{aligned}
& \frac{1}{1-u} L_{p, q: a_{1}}(u:-k, x) \\
= & \frac{1}{1-u} H_{k, \chi}^{(1)}\left(u, q \mid a_{1}\right)-\frac{\chi(p)[p: q]^{k}}{1-u^{p}} H_{k, \chi}^{(1)}\left(u^{p}, q^{p} \mid a_{1}\right) .
\end{aligned}
$$

Indeed,we see

$$
\begin{aligned}
& \int_{X^{*}}\left\langle a_{1} x: q\right\rangle^{k} \chi \omega^{k}(x) d \mu_{u}(x) \\
= & \int_{X} \chi(x)\left[a_{1} x: q\right]^{k} d \mu_{u}(x)-\chi(p)[p: q]^{k} \frac{1-u}{1-u^{p}} \int_{X}\left[a_{1} x: q^{p}\right]^{k} d \mu_{u^{p}}(x) .
\end{aligned}
$$

Since $\left|\left\langle a_{1} x: q\right\rangle-1\right|_{p}<p^{-\frac{1}{p-1}}$ for $x \in X^{*}$, we obtain

$$
\left\langle a_{1} x: q\right\rangle^{p^{n}} \equiv 1\left(\bmod p^{n}\right) .
$$

For $k \equiv k^{\prime}\left(\bmod (p-1) p^{n}\right)$, we have

$$
L_{p, q: a_{1}}\left(u:-k, \chi \omega^{k}\right) \equiv L_{p, q: a_{1}}\left(u:-k^{\prime}, \chi \omega^{k^{\prime}}\right)\left(\bmod p^{n}\right) .
$$




\section{References}

[1] T. Kim, An invariant $p$-adic Integral associated with Daehee numbers, Integral Transforms Spec. Funct. 13 (2002), 65-69.

[2] _ , p-adic $q$-integral associated with Changhee-Barnes' $q$-Bernoulli polynomials, Integral Transforms Spec. Funct. 15 (2004).

[3] Kummer Congruence for the Bernoulli numbers of higher order, Appl. Math. Comput. 151 (2004), 589-593.

[4] , q-Riemann Zeta functions, Int. J. Math. Math. Sci. 2004 (2004), no. 12, 599-605.

[5] - Analytic continuation of multiple q-Zeta functions and their values at negative integers, Russ. J. Math. Phys. 11 (2004), 71-76.

[6] $\longrightarrow$ On Euler-Barnes multiple zeta functions, Russ. J. Math. Phys. 10 (2003), 261-267.

[7] $\longrightarrow q$-Volkenborn integration, Russ. J. Math. Phys. 9 (2002), 288-299.

[8] - On p-adic $q$-L-functions and sums of powers, Discrete Math. 252 (2002), 179-187.

[9] - Some formulae for the $q$-Bernoulli and Euler polynomials of higher order, J. Math. Anal. Appl. 273 (2002), 236-242.

[10] — A note on q-multiple Zeta function, J. Physics 34 (2001), 643646.

[11]___ On p-adic q-Bernoulli numbers, J. Korean Math. Soc. 37 (2000), 27-30.

[12] _ A note on Dirichlet L-series, Proc. Jangjeon Math. Soc. 6 (2004), 161-166.

[13] _ A note on the q-analogue of multiple zeta function, Adv. Stud. Contemp. Math. 8 (2004), 111-113.

Leechae Jang, Department of Mathematics and Computer Science, KonKUK UNIVERSiTy, ChOONGJu 380-701, Korea

E-mail: leechae.jang@kku.ac.kr

Taekyun Kim, Institute of Science Education, Kongju National UniverSITY, KONGJU 314-701, Korea

E-mail: tkim@kongju.ac.kr 